\title{
Digestibilidade aparente de dietas e metabolismo de frangos de corte alimentados com dietas contendo soja integral processada
}

\author{
Apparent digestibility of the diets and metabolism of broilers fed with diets containing heat \\ processed full-fat soybean
}

\author{
Amanda d'Ávila Carvalho ${ }^{1}$ Irineo Zanella ${ }^{I^{*}}$ Cheila Roberta Lehnen ${ }^{\mathrm{I}}$ Ines Andretta ${ }^{\mathrm{III}}$ \\ Eloiza Lanferdini ${ }^{\mathrm{III}}$ Luciano Hauschild ${ }^{\mathrm{I}}$ Paulo Alberto Lovatto ${ }^{\mathrm{II}}$
}

RESUMO

Foi realizado um experimento para avaliar a digestibilidade aparente de dietas e o metabolismo de frangos de corte alimentados com dietas contendo soja integral (SI) processada a vácuo $\left(S I_{\text {vac }}\right)$ ou a vapor $\left(S I_{\text {vap }}\right)$. O delineamento experimental foi inteiramente casualizado, com cinco tratamentos (dieta controle - DC; DC com substituição isométrica de $40 \%$ de $S_{\text {vac }}$ ou $S I_{\text {vap }}$; dieta com $S_{\text {vac }}$ e dieta com $\left.S I_{\text {va }}\right)$. Os tratamentos com substituição isométrica avaliaram a digestibilidade das sojas processadas; os demais, a digestibilidade de dietas contendo ou não soja processada (controle). A ingestão de $N$ foi 23 e 20\% menor $(P<0,01)$ para as aves alimentadas com dietas com SI ou SI em relação ao grupo controle. A excreção, digestibilidade e absorção do $N$ não foram influenciadas $(P>0,05)$ pelas dietas. A excreção de energia foi 19 e $22 \%$ menor $(P<0,01)$ para as aves alimentadas com $S_{\text {vac }}$ na dieta em relação ao grupo controle $e$ àquele alimentado com dieta com $\mathrm{SI}_{\text {vap }}$. A dieta com $\mathrm{SI}$ melhorou $(P<0,01)$ a digestibilidade e a metabolização da energia. A energia metabolizável aparente corrigida para retenção de $N\left(E M_{n}\right)$ foi $12 \%$ superior $(P<0,01)$ à da dieta com $S I_{\text {vap }}$ e similar $(P<0,01)$ à da dieta controle. A matéria seca, a proteína e o extrato etéreo digestíveis, as energias metabolizável aparente e $E M_{n}$ foram semelhantes $(P>0,05)$ para a soja integral processada a vácuo ou a vapor. A soja processada a vácuo ou a vapor é uma alternativa ao farelo de soja e ao óleo vegetal em dietas para frangos de corte.

Palavras-chave: alimentos alternativos, avicultura, energia metabolizável, processamento.

\section{ABSTRACT}

An experiment was carried out to evaluate the apparent digestibility of diets and the metabolism of broilers fed diets containing full-fat soybean (FFS) processed by vacuum $\left(F_{F S}\right)$ or by steam (FFS $)$. The experimental design was completely randomized with five treatments (control diet $C D, C D$ with isometric substitution of $40 \% F_{\text {FF }}$ or FFS ; diet with FFS $_{v a c}$ and diet with $F F S_{\text {stm }}$ ). The treatments with isometric substitution evaluated the digestibility of processed soybeans; the remains, the digestibility of diets containing either processed soybean or not (control). The $N$ intake was 23 and $20 \%$ less $(P<0.01)$ to the broilers fed with diets containing FFS or FFS than control group. The excretion, digestibility and absorption of $N$ were not influenced $(P>0.05)$ by the diets. The excretion of energy was 19 and $22 \%$ less $(P<0.01)$ to the broilers fed with FFS in the diet than control group and those fed with FFS $S_{s t m}$ in the diet. The diet with FFS $S_{\text {vac }}$ enhanced $(P<0.01)$ the digestibility and the metabolization of energy. The apparent metabolizable energy corrected for $N$ retention $\left(M E_{n}\right)$ was $12 \%$ higher $(P<0.01)$ to the one fed of diet with FFS $_{\text {stm }}$ and similar to control diet $(P<0.01)$. The dry matter, protein and ether extract digestibles, the apparent metabolizable energy and $M E_{n}$ were similar $(P>0.05)$ for full-fat soybean processed by vacuum or by steam. Full-fat soybean processed by vacuum or by steam is an alternative to soybean meal and to the vegetal oil in the diets for broilers.

Key words: alternative feed, aviculture, metabolizable energy, processing.

\section{INTRODUÇÃO}

No Brasil, a alimentação de frangos de corte é baseada em dietas com milho e farelo de soja. A avicultura consome cerca de 49 e $24 \%$ da produção desses ingredientes, respectivamente (SINDIRAÇÕES, 2006). Nas últimas décadas, a taxa de crescimento do frango tem aumentado, estimulando a formulação de

IPrograma de Pós-graduação em Zootecnia (PPGZ), Departamento de Zootecnia (DZ), Universidade Federal de Santa Maria (UFSM), Santa Maria, RS, Brasil.

IIDZ, UFSM, 97105-900, Camobi, Santa Maria, RS, Brasil. E-mail: izanella@smail.ufsm.br. *Autor para correspondência.

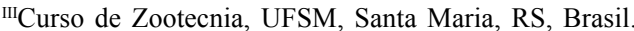


dietas mais densas energeticamente. Para o ajuste energético, o óleo vegetal é comumente utilizado, aumentando, contudo, o custo das rações.

A soja integral (SI) tem cerca de $20 \%$ de extrato etéreo e $36 \%$ de proteína, sendo uma alternativa ao farelo de soja e ao óleo vegetal. Esses teores permitem um melhor ajuste nutricional das dietas. Variações nos teores nutricionais, entretanto, ocorrem devido ao clima, ao solo, ao tipo de cultivo, à variedade genética e ao processamento.

A SI reduz o incremento calórico da dieta e apresenta maior relação custo-benefício no sistema produtivo (NITSAN et al., 1997; NRC, 1998; MATEOS et al., 2002). Estudos recentes têm indicado que os coeficientes de digestibilidade dos aminoácidos, do extrato etéreo e a energia metabolizável são superiores para a soja integral em relação ao farelo de soja mais óleo (CAFÉ et al., 2000ab). As substâncias antinutritivas, como inibidores de proteases, lectinas e oligossacarídeos, no entanto, limitam sua utilização in natura (LIENER, 2000). No sistema digestório das aves, a degradação dessas substâncias é parcial, reduzindo o aproveitamento do alimento.

Processos térmicos permitem a inativação das substâncias antinutritivas da soja. Dentre esses, pode-se citar a tostagem (em tambor rotativo ou jetsploder), a micronização, a extrusão e o uso de microondas. As técnicas mais recentes para o tratamento térmico da soja utilizam vapor combinado com vácuo ou unicamente vapor. Os processos com utilização de calor úmido têm indicado melhora na conservação e qualidade nutricional de produtos cárneos (BÖHM et al., 2006).

Vários estudos mostram que o valor nutritivo da SI para frangos de corte varia entre os tipos de processamento (ZANELLA et al., 1999; CAFÉ et al., 2000a; PALACIOS et al., 2004). Entretanto, as pesquisas com tratamentos de soja integral por processos a vácuo ou a vapor avaliando a digestibilidade e metabolismo de frangos de corte são poucas. O objetivo do estudo foi avaliar a digestibilidade e o valor energético da soja processada a vácuo ou a vapor e de dietas contendo soja processada a vácuo ou a vapor para frangos de corte.

\section{MATERIAL E MÉTODOS}

O experimento foi realizado no Laboratório de Análises Micotoxicológicas (LAMIC) da Universidade Federal de Santa Maria, de outubro a novembro de 2005. Foram utilizados 260 pintos, machos, linhagem Cobb, com peso médio inicial de 474 gramas. As aves foram alojadas em uma sala climatizada em 26 gaiolas de $0,5 \times 0,5 \times 0,4 \mathrm{~m}$, equipadas com comedouro e bebedouro tipo calha, campânula elétrica e bandeja para coleta de excretas. $\mathrm{O}$ delineamento experimental foi inteiramente casualizado, com cinco tratamentos (dieta controle - DC; DC com substituição isométrica de $40 \%$ de $\mathrm{SI}_{\text {vac }}$ ou $\mathrm{SI}_{\text {vap }}$; dieta com SI $\mathrm{vac}_{\text {vac }}$ e dieta com $\mathrm{SI}_{\text {vap }}$ ) e cada tratamento teve seis repetições com 10 aves, exceto para os tratamentos com substituição isométrica (quatro repetições). Os tratamentos com substituição isométrica avaliaram a digestibilidade das sojas processadas; os demais, a digestibilidade de dietas contendo ou não soja processada (controle).

$\mathrm{O}$ processamento a vácuo utilizado para a soja integral foi realizado em unidade industrial de desativação de soja através de aquecimento em reatores herméticos a $109^{\circ} \mathrm{C}$. Este processo combinou vácuo com pressão de $0,35 \mathrm{~atm}$ durante 18 minutos. Em seguida, os grãos foram resfriados a $35^{\circ} \mathrm{C}$, descascados e secados a $12 \%$ de umidade. O processamento a vapor para a soja integral foi realizado em desativadora modular com aquecimento por vapor a $106^{\circ} \mathrm{C}$ e pressão de $2 \mathrm{~atm}$ por 8 minutos. Após isso, os grãos foram secados a $12 \%$ de umidade.

As análises bromatológicas das sojas processadas foram realizadas conforme a AOAC (1990). A determinação de aminoácidos totais foi realizada com o equipamento NIR (Near Infrared Analysis), usando ajuste de calibração pelo procedimento analítico CEAN 010 da Adisseo Brasil SA (Tabela 1). As dietas experimentais (Tabela 2) foram formuladas utilizandose as recomendações nutricionais de ROSTAGNO et al. (2000) para frangos machos na fase inicial. Na formulação, foi atribuído o valor de energia metabolizável da soja integral tostada conforme ROSTAGNO et al. (2000).

O experimento teve duração de 10 dias, sendo cinco para adaptação ao alimento e manejo e cinco para coleta de excretas. Nesse período, o fornecimento de água e ração foi à vontade. Foi utilizado o método de coleta total de excretas com o início e fim determinados pelo aparecimento de excretas marcadas ( $1,0 \%$ de $\mathrm{Fe}_{2} \mathrm{O}_{3}$ adicionado às dietas). As excretas foram coletadas duas vezes ao dia, pesadas, acondicionadas em sacos plásticos e conservadas em congelador a $-10^{\circ} \mathrm{C}$. Ao final do experimento, foram homogeneizadas e amostradas $(0,4 \mathrm{~kg})$, secadas em estufa de ventilação forçada $\left(60^{\circ} \mathrm{C}\right.$ por $\left.72 \mathrm{~h}\right)$ e moídas para análises posteriores. Foram determinados a quantidade de ração consumida e o total de excretas produzido por unidade experimental. As análises químicas das dietas e excretas foram realizadas segundo metodologia da AOAC (1990).

Foram avaliados os coeficientes de digestibilidade aparente da matéria seca $\left(\mathrm{CD}_{\mathrm{a}} \mathrm{MS}\right)$, da 
Tabela 1 - Composição química da soja processada a vácuo ou a vapor ${ }^{\mathrm{a}}$.

\begin{tabular}{lcc}
\hline & \multicolumn{2}{c}{ Soja processada } \\
\cline { 2 - 3 } Item & Vácuo & Vapor \\
\hline Matéria seca, \% & 89,50 & 88,90 \\
Proteína bruta, \% & 40,68 & 36,59 \\
Energia bruta, kcal/kg & 5.456 & 5.178 \\
Extrato etéreo, \% & 21,41 & 21,24 \\
Fibra bruta, \% & 3,29 & 3,98 \\
Cinzas, \% & 4,58 & 4,87 \\
Cálcio, \% & 0,18 & 0,21 \\
Fósforo total, \% & 0,32 & 0,44 \\
$\quad$ Aminoácidos totais, \% & & \\
Arginina & 3,45 & 3,10 \\
Fenilalanina & 2,03 & 1,84 \\
Histidina & 1,06 & 0,93 \\
Isoleucina & 1,73 & 1,54 \\
Leucina & 3,21 & 2,67 \\
Lisina & 2,35 & 2,06 \\
Metionina & 0,47 & 0,37 \\
Met + Cis & 1,12 & 0,94 \\
Treonina & 1,51 & 1,32 \\
Triptofano & 0,51 & 0,44 \\
Valina & 1,83 & 1,69 \\
\hline
\end{tabular}

${ }^{\mathrm{a}}$ Matéria natural.

proteína bruta (CD PB), do extrato etéreo (CD EE) e pela energia bruta $(\mathrm{C} \mathrm{C} \mathrm{EB})$, o balanço do $\mathrm{N}$ e energias metabolizável aparente e corrigida para retenção de $\mathrm{N}$, conforme MATTERSON et al. (1965). Os dados obtidos foram submetidos à análise de variância pelo procedimento GLM (General Linear Model), incluindo no modelo os efeitos do tipo de processamento da soja. As análises estatísticas foram realizadas através do software Minitab (MCKENZIE \& GOLDMAN, 1999). As diferenças entre as médias foram comparadas pelo Teste de Tukey a 5\% de probabilidade.

\section{RESULTADOS E DISCUSSÃO}

A composição química da soja processada a vácuo e a vapor é apresentada na tabela 1. Para os tipos de processamento de soja estudados, não foram encontradas informações da composição nutricional em tabelas mais recentes de composição de alimentos (NRC, 1998; ROSTAGNO et al., 2000). Neste estudo, foram observadas pequenas variações na composição química dos tipos de soja estudados. Essa composição pode ser influenciada por fatores como solo, clima, cultivar e processamento da soja integral (FISCHER JR. et al., 1998).
$\mathrm{O}$ balanço do $\mathrm{N}$ de frangos de corte e o controle de qualidade das sojas processadas estão apresentados na tabela 3 . A ingestão de $\mathrm{N}$ foi influenciada pelas dietas $(\mathrm{P}<0,01)$, sendo utilizada como covariável para o ajuste das demais respostas. As aves alimentadas com SI ${ }_{\text {vac }}$ ou SI nap dieta ingeriram $23 \mathrm{e}$ $20 \%$ menos $\mathrm{N}(\mathrm{P}<0,01)$ em relação ao grupo controle. A digestão e absorção de $\mathrm{N}$ não foram influenciadas $(\mathrm{P}>0,05)$ pelo processamento da soja. Para o controle de qualidade, a atividade ureática (AU) da $\mathrm{SI}_{\text {vac }}$ e a solubilidade protéica (SP) da SI vap estiveram dentro dos limites recomendados (ANFAR, 1985; ARABA \& DALE, 1990). Ao contrário, a SP para a SI vac $(89,29 \%)$ e a AU para a SIvap $(0,03$ ÄpH) indicaram sub e superaquecimento, respectivamente.

Alguns diluentes inertes utilizados em dietas, como areia ou caulim, podem originar sinais de saciedade em aves (LABIER \& LECHERQ, 1994). Isso pode explicar a menor ingestão de $\mathrm{N}$ das aves alimentadas com dietas com SIvac ou SIvap, conseqüência do menor consumo de matéria seca.

A AU e a SP são critérios utilizados na avaliação da eficácia do tratamento térmico da soja. Para a soja, o valor aceitável de AU varia de 0,05 a 0,3 ÄpH, mas não há definição na literatura quanto ao índice ideal (FRIESEN et al., 1993). Além disso, a AU é pouco eficaz na avaliação de sojas superaquecidas, não sendo um indicador seguro da qualidade nutricional. A SP avalia o grau de desnaturação da proteína e a biodisponibilidade de aminoácidos. Valores acima de $85 \%$ sugerem subaquecimento e abaixo de $70 \%$ superaquecimento do farelo ou grãos de soja (ARABA \& DALE, 1990). Mesmo que a SP para a $\mathrm{SI}_{\text {vac }}$ e a AU para a $\mathrm{SI}_{\text {vap }}$ tenham indicado sub e superaquecimento da soja, respectivamente, o balanço do $\mathrm{N}$ foi semelhante. Isso pode ser explicado em parte pelo curto período experimental, que não foi suficiente para alterar o balanço do nitrogênio. Além disso, os valores de AU e SP adequados segundo a literatura são aplicados a processos com uso de calor seco. É provável que esses padrões não sejam aplicáveis para tecnologias com utilização de calor úmido, como nos processos a vácuo e a vapor.

Os resultados de ingestão, excreção, digestibilidade da energia bruta e energias metabolizável aparente $\left(\mathrm{EM}_{\mathrm{a}}\right)$ e corrigida para retenção de $\mathrm{N}\left(\mathrm{EM}_{\mathrm{n}}\right)$ das dietas contendo soja processada a vácuo ou a vapor são apresentados na tabela 4 . A ingestão de energia não foi influenciada $(\mathrm{P}>0,05)$ pelo tipo de processamento. A excreção, o coeficiente de digestibilidade, a EM $\left(\mathrm{kcal} \mathrm{kg}^{-1} \mathrm{MS}\right)$ e $\mathrm{EM}_{\mathrm{n}}\left(\mathrm{kcal} \mathrm{kg}^{-1}\right.$ MS) diferiram entre os tratamentos. As aves alimentadas com dieta contendo $\mathrm{SI}_{\mathrm{vac}}$ excretaram $19 \mathrm{e}$ 
Tabela 2 - Ingredientes, composição calculada e analisada das dietas experimentais.

\begin{tabular}{|c|c|c|c|}
\hline \multirow{2}{*}{ Ingredientes, \% } & \multicolumn{3}{|c|}{ Dietas } \\
\hline & Controle & $\mathrm{SI}_{\mathrm{vac}}$ & $\mathrm{SI}_{\text {vap }}$ \\
\hline Milho & 53,53 & 38,73 & 31,06 \\
\hline Farelo de soja & 38,82 & 0,00 & 0,00 \\
\hline Soja vácuo & 0,00 & 51,11 & 0,00 \\
\hline Soja vapor & 0,00 & 0,00 & 58,91 \\
\hline Óleo vegetal & 3,59 & 0,00 & 0,00 \\
\hline Calcário & 0,90 & 0,89 & 1,05 \\
\hline Fosfato bicálcico & 1,91 & 2,02 & 2,16 \\
\hline Cloreto de sódio & 0,40 & 0,40 & 0,40 \\
\hline DL-Metionina 99\% & 0,18 & 0,10 & 0,09 \\
\hline L-Lisina 99\% & 0,06 & 0,00 & 0,04 \\
\hline L-treonina $98,5 \%$ & 0,11 & 0,08 & 0,03 \\
\hline Suplemento vitamínico e mineral ${ }^{\mathrm{a}}$ & 0,50 & 0,50 & 0,50 \\
\hline Inerte $^{\mathrm{b}}$ & 0,00 & 6,18 & 5,75 \\
\hline \multicolumn{4}{|l|}{ Valores calculados } \\
\hline Energia metabolizável, kcal/kg & 3.000 & 3.000 & 3.000 \\
\hline Proteína bruta, \% & 21,40 & 21,40 & 21,40 \\
\hline Cálcio, \% & 0,96 & 0,96 & 0,96 \\
\hline Fósforo disponível, \% & 0,45 & 0,45 & 0,45 \\
\hline Lisina, $\%$ & 1,14 & 1,16 & 1,14 \\
\hline Metionina, $\%$ & 0,59 & 0,43 & 0,49 \\
\hline \multicolumn{4}{|l|}{ Valores analisados ${ }^{\mathrm{c}}$} \\
\hline Matéria seca, \% & 88,20 & 89,46 & 89,45 \\
\hline Proteína bruta, \% & 25,85 & 21,63 & 21,49 \\
\hline Extrato etéreo, \% & 3,46 & 9,46 & 9,82 \\
\hline Fósforo total, \% & 0,58 & 0,68 & 0,69 \\
\hline Energia bruta, $\mathrm{kcal} / \mathrm{kg}$ & 4.482 & 4.535 & 4.269 \\
\hline
\end{tabular}

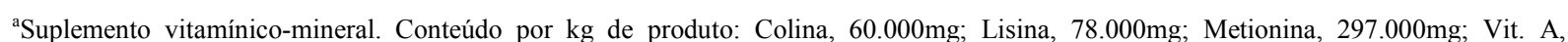
2.200.000 UI; Vit. D3, 500.000 UI; Vit. E, 5.000mg; Vit. K3, 660mg; Vit. B1, 440mg; Vit. B2, 1.150mg; Vit. B6, 926mg; Vit. B12, 3.600mcg; Biotina, 36mg; Ác. Fólico, 250mg; Ác. Nicotínico, 5.560mg; Ác. Pantotênico, 3.600mg; Coccidiostático, 12.000mg; Ferro, 9.998mg; Cobre, 1.600mg; Zinco, 10.996mg; Manganês, 11.993mg; Iodo, $88 \mathrm{mg}$; Selênio, 40mg; Promotor de crescimento e eficiência alimentar, $1.000 \mathrm{mg}$.

${ }^{\mathrm{b} C}$ Caulim.

${ }^{\mathrm{c}}$ Matéria natural.

$22 \%$ menos energia $(\mathrm{P}<0,01)$ em relação ao grupo controle e ao alimentado com dieta contendo SI vap. Isso possivelmente contribuiu para o melhor resultado de digestibilidade da energia bruta da dieta com $\mathrm{SI}_{\text {vac }}$ em relação às demais. Do mesmo modo, para $\mathrm{a} \mathrm{EM}_{\mathrm{a}}$, a dieta com $\mathrm{SI}_{\text {vac }}$ obteve melhor resultado $(\mathrm{P}<0,01)$. A EM para as aves alimentadas com $\mathrm{SI}_{\text {vac }}$ foi $12 \%$ superior $(\mathrm{P}<0,01)$ em relação ao tratamento com $\mathrm{SI}_{\text {vap }}$ e similar ao grupo controle.

As diferenças obtidas no balanço energético de animais alimentados com dietas com soja integral processada estão relacionadas à composição química dos grãos (LÁZARO et al., 2002). Há correlação positiva entre a energia bruta da soja e a energia disponível para os processos metabólicos das aves (ZONTA et al., 2006). No presente estudo, a SI apresentou $4,4 \%$ mais energia bruta que a SI vap $(6.096$ vs $5.825 \mathrm{kcal} \mathrm{kg}^{-1} \mathrm{MS}$ ). Isso pode ter influenciado na energia metabolizável aparente da dieta com $\mathrm{SI}_{\text {vac }}$, a qual foi $11,3 \%$ superior em relação à da dieta com SI $\mathrm{SI}_{\text {var. }}$. A remoção da casca da soja no processo a vácuo pode ter melhorado a metabolizabilidade da energia. Como a digestão enzimática da fibra é limitada em frangos de corte, em processos com extração desse componente há melhora no valor energético (MATEOS et al., 2002).

Em frangos de corte, cerca de $8 \mathrm{kcal} \mathrm{g}^{-1}$ de $\mathrm{N}$ são retidas pela oxidação do ácido úrico (HILL \& ANDERSON, 1958). Esse valor é utilizado para o ajuste do balanço do $\mathrm{N}$ na determinação da $\mathrm{EM}$. Em aves jovens, a relação entre ingestão e excreção de $\mathrm{N}$ é diretamente proporcional (ISHIBASHI \& YONEMOCHI, 2002). Neste estudo, as aves alimentadas com a dieta controle ingeriram e, consequentemente, excretaram mais nitrogênio. A similaridade na energia metabolizável 
Tabela 3 - Balanço do nitrogênio de frangos de corte alimentados com dietas contendo soja processada a vácuo ou a vapor e controle de qualidade das sojas processadas.

\begin{tabular}{|c|c|c|c|c|c|}
\hline \multirow{2}{*}{ Variáveis } & \multicolumn{3}{|c|}{ 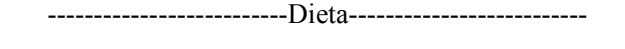 } & \multirow{2}{*}{$\mathrm{CV}, \%^{\mathrm{d}}$} & \multirow{2}{*}{$\mathrm{P}^{\mathrm{e}}$} \\
\hline & Controle & $\mathrm{SI}_{\mathrm{vac}}$ & $\mathrm{SI}_{\text {vap }}$ & & \\
\hline \multicolumn{6}{|l|}{ Nitrogênio } \\
\hline Ingerido, $\mathrm{g} / \mathrm{dia}^{\mathrm{f}}$ & $26,76^{\mathrm{a}}$ & $20,71^{\mathrm{b}}$ & $21,42^{\mathrm{b}}$ & 13,95 & 0,000 \\
\hline Excreta, g/dia & 7,81 & 5,46 & 5,83 & 25,57 & 0,179 \\
\hline Coeficiente de digestibilidade, $\%$ & 71,15 & 73,68 & 72,79 & 5,20 & 0,157 \\
\hline Absorvido, g/dia & 18,95 & 15,25 & 15,60 & 11,66 & 0,177 \\
\hline \multirow{2}{*}{ Controle de qualidade } & \multicolumn{5}{|c|}{ Critérios } \\
\hline & \multicolumn{2}{|c|}{ Atividade ureática, $\Delta \mathrm{pH}$} & \multicolumn{3}{|c|}{ Solubilidade protéica, $\%$} \\
\hline $\mathrm{SI}_{\text {vac }}$ & \multicolumn{2}{|c|}{0,12} & \multicolumn{3}{|c|}{89,29} \\
\hline $\mathrm{SI}_{\text {vap }}$ & \multicolumn{2}{|c|}{0,03} & \multicolumn{3}{|c|}{74,50} \\
\hline
\end{tabular}

a, b, c Letras diferentes na mesma linha diferem pelo Teste de Tukey $(\mathrm{P}<0,01) ;{ }^{\mathrm{d}}$ coeficiente de variação; ${ }^{\mathrm{e}}$ nível de $5 \%$ de significância; utilizado como covariável.

das dietas controle e com SI vac evidencia esse ajuste, pois a maior excreção de $\mathrm{N}$ pelo grupo controle influenciaria a $\mathrm{EM}_{\mathrm{n}}$.

Os resultados de matéria seca, proteína e extrato etéreo digestíveis, energias metabolizável aparente e aparente corrigida para $\mathrm{N}$ da soja integral processada a vácuo ou a vapor estão apresentados na tabela 5. O tipo de processamento da soja não influenciou $(\mathrm{P}>0,05)$ as variáveis estudadas.

O processamento de alimentos pelo calor é utilizado para a conservação de carnes, grãos, frutas e vegetais (DROUZAS \& SCHUBERT, 1996). Até a década de 80 , as tecnologias de processamento disponíveis se baseavam no uso de calor seco (KOZANOGLU et al., 2006). Com essa técnica, o aquecimento é desuniforme, comprometendo o valor nutritivo da soja (PRACHAYAWARAKORN et al.,
2006). Atualmente, processos que utilizam vapor ou sua combinação com vácuo têm surgido para assegurar a qualidade física e nutricional dos grãos. Esses processos podem favorecer o rompimento de ligações químicas nos nutrientes que dificultam a ação enzimática no trato gastrintestinal do frango de corte.

No presente estudo, considerando a dieta, a digestibilidade da energia bruta, EM e EM melhoraram com a inclusão de soja processada a vácuo em relação à processada a vapor. Esse efeito não foi observado para as variáveis da digestão e metabolismo dos nutrientes dessa matéria-prima. Para estimar essas variáveis, substitui-se parte da dieta controle (20 a 40\%) pelo ingrediente-teste. Esse procedimento leva a um desbalanceamento da dieta. Nutrientes em excesso, como a proteína, podem gerar erros na determinação do valor nutritivo (JESUS PEREIRA et al., 2004). É

Tabela 4 - Consumo, digestibilidade e metabolização da energia de dietas contendo soja processada a vácuo ou a vapor para frangos de corte.

\begin{tabular}{|c|c|c|c|c|c|}
\hline \multirow{2}{*}{ Variáveis } & \multirow[b]{2}{*}{ Controle } & -Dieta--- & \multirow[b]{2}{*}{$\mathrm{SI}_{\text {vap }}$} & \multirow{2}{*}{$\mathrm{CV} \%{ }^{\mathrm{d}}$} & \multirow{2}{*}{$\mathrm{P}^{\mathrm{e}}$} \\
\hline & & $\mathrm{SI}_{\mathrm{vac}}$ & & & \\
\hline \multicolumn{6}{|l|}{ Animal } \\
\hline Energia ingerida, $\mathrm{kcal} \mathrm{dia}^{-1}$ & 2900 & 2714 & 2659 & 7,96 & 0,135 \\
\hline Energia na excreta, $\mathrm{kcal}_{\mathrm{dia}}{ }^{-1}$ & $550^{\mathrm{a}}$ & $444^{\mathrm{b}}$ & $567^{\mathrm{a}}$ & 14,33 & 0,002 \\
\hline $\mathrm{EM}_{\mathrm{a}}, \mathrm{kcal} \mathrm{dia}^{-1}$ & 2350 & 2270 & 2091 & 9,04 & 0,067 \\
\hline $\mathrm{EM}_{\mathrm{n}}, \mathrm{kcal} \mathrm{dia}^{-1}$ & 2195 & 2144 & 1963 & 9,17 & 0,084 \\
\hline \multicolumn{6}{|l|}{ Dieta } \\
\hline Coeficiente de digestibilidade, $\%$ & $81,06^{\mathrm{a}}$ & $83,55^{\mathrm{b}}$ & $78,67^{\mathrm{c}}$ & 3,07 & 0,000 \\
\hline $\mathrm{EM}_{\mathrm{a}}, \mathrm{kcal} \mathrm{kg}^{-1} \mathrm{MS}^{\mathrm{f}}$ & $4119^{\mathrm{a}}$ & $4235^{\mathrm{b}}$ & $3754^{\mathrm{c}}$ & 5,50 & 0,000 \\
\hline $\mathrm{EM}_{\mathrm{n}}, \mathrm{kcal} \mathrm{kg}^{-1} \mathrm{MS}^{\mathrm{g}}$ & $3845^{\mathrm{a}}$ & $4000^{\mathrm{a}}$ & $3524^{\mathrm{b}}$ & 5,68 & 0,000 \\
\hline
\end{tabular}

${ }^{\mathrm{a}, \mathrm{b}, \mathrm{c}}$ Letras diferentes na mesma linha diferem pelo Teste de Tukey $(\mathrm{P}<0,05) ;{ }^{\mathrm{d}}$ coeficiente de variação; ${ }^{\mathrm{e}}$ nível de $5 \%$ de significância; ${ }^{\mathrm{f}}$ energia metabolizável aparente, ${ }^{\mathrm{g}} \mathrm{EM}_{\mathrm{a}}$ corrigida para retenção de $\mathrm{N}$.

Ciência Rural, v.38, n.2, mar-abr, 2008. 
Tabela 5 - Matéria seca, proteína, extrato etéreo e energia digestíveis, energia metabolizável e controle de qualidade da soja processada a vácuo ou a vapor.

\begin{tabular}{|c|c|c|c|c|}
\hline \multirow{2}{*}{ Variáveis } & \multicolumn{2}{|c|}{----------Soja processada---------- } & \multirow{2}{*}{$\mathrm{CV} \%{ }^{\mathrm{a}}$} & \multirow{2}{*}{$\mathrm{P}^{\mathrm{b}}$} \\
\hline & Vácuo & Vapor & & \\
\hline \multicolumn{5}{|l|}{ Nutrientes digestíveis, $\%$} \\
\hline Matéria seca digestível & 54,63 & 50,31 & 12,91 & 0,408 \\
\hline Proteína digestível & 34,10 & 36,18 & 3,16 & 0,298 \\
\hline Extrato etéreo digestível & 21,43 & 20,82 & 2,59 & 0,121 \\
\hline $\mathrm{EM}_{\mathrm{a}}, \mathrm{kcal} / \mathrm{kg} \mathrm{MS}$ & 3375 & 3045 & 13,14 & 0,302 \\
\hline $\mathrm{EM}_{\mathrm{n}}, \mathrm{kcal} / \mathrm{kg} \mathrm{MS}$ & 3257 & 3012 & 11,35 & 0,368 \\
\hline
\end{tabular}

${ }^{a}$ Coeficiente de variação; ${ }^{b}$ nível de $5 \%$ de significância; ${ }^{c}$ energia metabolizável aparente, ${ }^{\mathrm{d}} \mathrm{EM}_{\mathrm{a}}$ corrigida para retenção de $\mathrm{N}$.

provável que, no presente experimento, o nível do ingrediente-teste utilizado tenha sido elevado. Isso, associado à idade dos frangos de corte (16 dias), contribuiu para a não diferença na digestão e no metabolismo das sojas processadas.

\section{CONCLUSÕES}

A soja processada a vácuo melhora a digestibilidade da energia bruta e a energia metabolizável aparente de dietas para frangos de corte. A digestibilidade das dietas e a absorção do nitrogênio em frangos de corte são semelhantes com o uso de soja integral processada a vácuo ou a vapor.

Entre os tipos de soja estudados, não há diferenças quanto à matéria seca, à proteína e ao extrato etéreo digestíveis, e às energias metabolizável aparente e corrigida para retenção de nitrogênio. A soja processada a vácuo ou a vapor é uma alternativa ao farelo de soja e ao óleo vegetal em dietas para frangos de corte.

\section{AGRADECIMENTOS}

Os autores agradecem às empresas Delta Indústria de Equipamentos Agroindustriais Ltda e SanLac Panapharm e também ao LAMIC, pelo apoio na realização do experimento. À Comissão de Aperfeiçoamento de Pessoal de Nível Superior (CAPES), pela concessão de bolsa à doutoranda do Programa de Pós-graduação em Zootecnia (PPGZ) Amanda d'Ávila Carvalho. Ao Conselho Nacional de Desenvolvimento Científico e Tecnológico $(\mathrm{CNPq})$, pela concessão de bolsa à graduanda Ines Andretta. À Pró-reitoria de Assuntos Estudantis (PRAE), pela concessão de bolsa à graduanda Eloiza Lanferdini.

\section{REFERÊNCIAS}

ANFAR. Associação nacional dos fabricantes de rações. Matérias-primas para alimentação animal - Padrão ANFAR. 4.ed. São Paulo, 1985. 65p
AOAC. Official methods of analysis. 15.ed. Arlington, VA, 1990. 1200p.

ARABA, M.; DALE, N.M. Evaluation of protein solubility as an indicator of overprocessing of soybean meal. Poultry Science, v.69, p.76-83, 1990.

BÖHM, V. et al. Improving the nutritional quality of microwavevacuum dried strawberries: a preliminary study. Food Science Technology International, v.12, p.67-75, 2006.

CAFÉ, M.B. et al. Determinação do valor nutricional das sojas integrais processadas para aves. Revista Brasileira de Ciência Avícola, v.2, n.1, p.67-74, 2000 b.

CAFÉ, M.B. et al. Composição e digestibilidade dos aminoácidos das sojas integrais processadas para aves. Revista Brasileira de Ciência Avícola, v.2, n.1, p.59-66, 2000a.

DROUZAS, A.E.; SCHUBERT, H. Microwave application in vacuum drying of fruits. Journal of Food Engineering, v.28, p.203-209, 1996.

FISCHER JR., A.A. et al. Determinação dos valores de energia metabolizável de alguns alimentos usados na alimentação de aves. Revista Brasileira de Zootecnia, v.27, p.314-318, 1998 .

FRIESEN, K.G. et al. The effect of moist extrusion of soy products on growth performance and nutrient utilization in the early-weaned pig. Journal Animal Science, v.71, p.2099$2109,1993$.

HILL, F.W.; ANDERSON, D.L. Comparison of metabolizable energy and produtive energy determinations with growing chicks. Journal Nutrition, v.64, p.587-604, 1958.

ISHIBASHI, T.; YONEMOCHI, C. Possibility of amino acid nutrition in broiler. Animal Science Journal, v.73, p.155165,2002 .

JESUS PEREIRA, L.E.D. et al. Digestibilidade de alimentos protéicos para suínos com diferentes dietas-referência. Boletim de Indústria Animal, v.61, p.75-81, 2004. 
KOZANOGLU, B. et al. Drying of seeds in a superheated steam vaccum fluidized bed. Journal of Food Engineering, v. 75, p. 383-387, 2006.

LABIER, M.; LECHERQ, B. Nutrition and feeding of poultry. Leicestershire: Nottingham University, 1994. 305p.

LÁZARO, R. et al. Whole soybeans in diets for poultry. 2002. Capturado em 25 de julho de 2006. On line. Disponível em: http://www.asa-europe.org/Library/library_e.htm\#Feed.

LEESON, S.; SUMMERS, J.D. Commercial poultry nutrition. Guelph: University Mooks, 1997. 350p.

LIENER, I.E. Non-nutritive factors and bioactive compounds in soy. In: Soy in animal nutrition. Savoy: Fed Anim Sci Soc, 2000. p.13-45.

MATEOS, G.G. et al. Traitement de la graine de soja 2002. Capturado em 20 de fevereiro de 2006. On line. Disponível em: http://www.asa-europe.org/pdf/processsb_f.pdf.

MATTERSON, L.D. et al. The metabolizable energy of feed ingredients for chickens. Storrs: University of Connecticut, 1965. v.11, p.11. (Agricultural Experiment Station Research Report).

MCKENZIE, J.; GOLDMAN, R.N. The student edition of minitab for windows manual. Softcover ed. Belmont Addison-Wesley Longman, Incorporated, 1999. v.12, p.592.

NITSAN, Z. et al. Effect of added soyabean oil and dietary energy on metabolisable and net energy of broiler diets. British Poultry Science, v.38, p.101-106, 1997.

NRC. NATIONAL RESEARCH COUNCIL. Nutrient requirements of swine. 10.ed. Washington, DC.: National Academy, 1998. 189p.

PALACIOS, M.F. et al. Effect of soybean variety and processing on growth performance of young chicks and pigs. Journal Animal Science, v.82, p.1108-1114, 2004.

PRACHAYAWARAKORN, S. et al. Heating process of soybean using hot-air and superheated-steam fluidized-bed dryers. LWT, v.39, p.770-778, 2006.

ROSTAGNO, H.S. et al. Tabelas brasileiras para aves e suínos: composição de alimentos e exigências nutricionais. Viçosa, MG: Universidade Federal de Viçosa, 2000. $141 \mathrm{p}$

SINDIRAÇÕES. Sindicato nacional da indústria da alimentação animal. 2006. Capturado em 20 de fevereiro de 2006. On line. Disponível em: www.sindiracoes.org.br.

ZANELLA, I. et al. Effect of enzyme supplementation of broiler diets based on corn and soybeans. Poultry Science, v.78, p.561-568, 1999.

ZONTA, M.C.D.M. et al. Energia metabolizável de farinhas de soja ou produtos de soja, determinada pelo método de coleta total e por equações de predição. Arquivos de Zootecnia, v. 55, p. $21-30,2006$ 